\title{
Propuesta de una aplicación interactiva con tecnología de códigos QR de la flora y fauna del Parque Biotemático Megua
}

\author{
María Fernanda Gutiérrez Polo ${ }^{\mathrm{a}}$, Aura Cristina Russill Meza ${ }^{\mathrm{a}}$, Iader Lamilla ${ }^{\mathrm{b}}$ \& Meglys Pérez Bernal ${ }^{\mathrm{a}}$ \\ ${ }^{a}$ Ingeniería Electrónica y Telecomunicaciones, Universidad Autónoma del Caribe, Barranquilla, Colombia. \\ ${ }^{b}$ Parque Biotemático Megua, Galapa, Colombia. \\ maría.gutierrez14@uac.edu.co, aura.russill@uac.edu.co,meglys.perez@uac.edu.co \\ iaderlamilla@gmail.com
}

Recibido: Septiembre 07, 2020.

Recibido en su versión corregida: Septiembre 29, 2020.

Aceptación: Octubre 16, 2020.

Cómo citar: Gutiérrez Polo, M F., Russill Meza, A.C., Escamilla, I. \& Pérez Bernal, M. (2020). Propuesta de una aplicación interactiva con tecnología de códigos QR de la flora y fauna del Parque Biotemático Megua.. Revista Sextante, 23 , pp. 37 - 47, 2020.

\section{Resumen}

Se presenta la propuesta del desarrollo de una aplicación móvil académica que permite la interacción con las especies de fauna y flora que habitan en el Parque Biotemático Megua. Esta reserva natural se encuentra ubicada en el Km 14 vía la Cordialidad Galapa (Atlántico) y su misión es educar y sensibilizar a los visitantes sobre el medio ambiente. La aplicación utiliza la lectura de códigos QR, los cuales son escaneados con la cámara de un dispositivo móvil, de esta manera se pueda obtener la información de diversas especies de flora y fauna que vivan en el Parque. Adicional, se desea presentar un juego académico; tipo cuestionario, que cuente con un banco de preguntas aleatorias, que permita a los visitantes poner a prueba los conocimientos adquiridos durante el recorrido. Como consecuencia de lo antes mencionado, el objetivo que se aspira alcanzar es hacer más interactiva e innovadora la visita al Parque, atrayendo más visitantes.

Palabras claves: Aplicación; Código QR; Fauna; Flora; Innovación; Megua.

\section{Proposal of an interactive application with QR code technology of the flora and fauna of the Parque Biotemático Megua}

\begin{abstract}
The proposal is presented for the development of an academic mobile application that allows interaction with the species of fauna and flora that inhabit the Parque Biotemático Megua. This nature reserve is located at Km 14 via the Cordiality Galapa (Atlántico) and its mission is to educate and sensitize visitors about the environment. The application uses the reading of QR codes, which are scanned with the camera of a mobile device, in this way can obtain the information of various species of flora and fauna that live in the Park. Additionally, incorporate an academic game afterward or in future works; questionnaire type, which has a bank of random questions, that allows visitors to test the knowledge acquired during the tour. As a result of the above, the aim is to make the visit to the Park more interactive and innovative, attracting more visitors. This article analyzes the implementation of these policies by the National Navy, through the actions of the Marine Corps in Montes de María, and the military victory that led to obtaining military control in the area.
\end{abstract}

Keywords: Application; QR code; Fauna; Flora; Innovation; Megua. 


\section{Introducción}

Generalmente, los parques temáticos suelen invertir un fuerte capital en nuevas tecnologías para crear un espacio innovador y llamativo a los turistas $\mathrm{y}$ visitantes para generar mayores ingresos $\mathrm{y}$ posicionar dicho establecimiento.

Aquellos que no se incorporan en lo antes mencionado, corren el riesgo de no tener un público suficiente para poder subsistir económicamente quedando rezagados y posiblemente olvidados.

El Parque Biotemático Megua es una reserva natural ubicada en el Km 14 vía la Cordialidad Galapa (Atlántico), cuya misión es educar y sensibilizar a los visitantes acerca del medio ambiente, involucrándolos con la biodiversidad natural y educándolos para el cuidado del medio ambiente.

Hoy en día, las visitas al Parque generan ingresos que permiten el escaso sostenimiento de este, impidiéndole incursionar en el desarrollo de nuevas tecnologías que proporcionen nuevas experiencias al público.

Adicional a lo anterior, la información y conocimiento que el Parque suministra al público que los visita es manejada directamente por los guías que proveen el recorrido, sin poder tener acceso de ninguna otra forma a ella de manera física y/o virtual, restringiéndola, debido a que el parque no cuenta con los bienes para implementar nuevas formas de compartir y comunicar sus conocimientos.

Por último, adjunto al impedimento de inversión de tecnología innovadora, se encuentra el hecho de que no se proporciona la suficiente publicidad, para que el Parque cuente con un mayor flujo de público visitante, recayendo nuevamente a la carencia de capital para el sostenimiento de este.

Actualmente, una gran cantidad de parques internacionales y nacionales, temáticos, naturales y culturales usan la tecnología para innovar sus establecimientos y hacerlos más atractivos. Como resultado obtienen un reconocimiento público, mejor manejo de la información y beneficios económicos para ellos.
Entre los parques internacionales se encuentra en Lodhi Gardens en Nueva Delhi (Shandilya, 2019), en el cual se asignaron a 100 árboles códigos QR escaneables con teléfonos inteligentes, los cuales arroja información sobre los árboles como la edad, vida útil, nombre, tiempo de floración, entre otros, con la finalidad de que el paseo por el parque sea más agradable e informativo.

A nivel nacional, en la ciudad de Ibagué (Tolima), la Corporación Autónoma Regional del Tolima (Cortolima); (CARACOL RADIO, 2020) lanzó un programa piloto para la marcación de los árboles del casco urbano, comenzando con el censo de 240 árboles existente en la sede principal de Cortolima, marcándolos con un Código QR. Su propósito es que la información técnica de la especie se pueda leer o divisar en cualquier dispositivo móvil, con el objetivo de replicarlo en todas las zonas verdes de la ciudad y se dé a conocer la importancia de los servicios ecosistémicos y ambientales.

Debido a esto, se presenta una propuesta para ser implementada en el Parque Biotemático Megua, la cual brinde a sus visitantes una experiencia diferente con el uso de la tecnología y le permita al Parque mayor visualización y posicionamiento a nivel regional.

Esto podría generar un incremento en el flujo de turistas, quienes tendrían acceso a la información de las especies que habitan en el Parque y se logre la misión de educarlos ecológicamente. Un mayor flujo de visitantes trae consigo el beneficio o incremento económico para la sustentación del Parque y la mejora continua de la innovación en el mismo.

A continuación, se muestra la sección correspondiente a la metodología y posterior a esta, la división de resultados y discusiones, conclusión y un apartado de agradecimiento.

\section{Metodología}

Se lleva a cabo diferentes etapas en el desarrollo de la propuesta: 
2.1. Levantamiento de la información y desarrollo de inventario de las especies existentes de fauna $y$ flora del Parque Biotemático Megua

La recolección de información se inicializó con las salidas de campo hechas por las estudiantes Aura Russill y María Fernanda Gutiérrez entre los meses de noviembre y diciembre del 2019.

Se recolectó la información de la fauna y flora del Parque a través de un inventario físico el cual fue digitalizado en ofimática con posterioridad; el señor Iader Lamilla, director del Parque, brindó la información faltante que permitió completar el trabajo realizado y recolectado. En la Figura 1, se presenta un collage con fotos de alguna de las especies que se encuentran en el Parque.

\subsection{Construcción de la estructura y parametrización de las plantillas sobre la información de flora y fauna del Parque Biotemático Megua:}

Se realizó una capacitación del parque por parte del señor Iader lamilla, director de éste, que además es Ingeniero Ambiental, Especialista en la Planeación para la Educación Ambiental y Magister en Auditoria en Tecnología e Ingeniería Ambiental. Esta capacitación fue realizada en relación con los parámetros adecuados que deben tener las especies para conservarse en el ecosistema. Adicionalmente, se realizó la sugerencia de agregar a las plantillas el parámetro de "hechos interesantes" acerca de las especies, para hacerlo más cautivador al lector.

A continuación, se publica el formato inicial en Word de cómo están parametrizadas las plantillas de flora y fauna, siendo también parte de la ellas la Figura 2 y $\underline{3}$ correspondiendo respectivamente a la especie herbal de la ruda y el animal o pájaro guacamaya.

\section{Ruda}

Nombre científico: Ruta graveolens.

Descripción: Es una planta sub-arbustiva que cuenta con muchas ramificaciones y con el tiempo su base es semi- leñosa a leñosa, es aromática y perenne, es decir, que perdura con el tiempo. Su altura varía entre los 40 a $100 \mathrm{~cm}$; Sus hojas, algo carnosas y de color verde glauco, o verde azuladas, son alternas, bi-o tripinnadas, esto se refiere a que cada foliolo (hoja) se articulan en un tallo central formando ángulo recto y en numerosas ramificaciones; con folios (hojas) oblongos (más largas que anchas) o espatulados. Su flor es un corimbo, con pequeñas flores de cuatro o cinco pétalos amarillos y sus frutos son capsulas redondas, su sabor es un poco amargo.

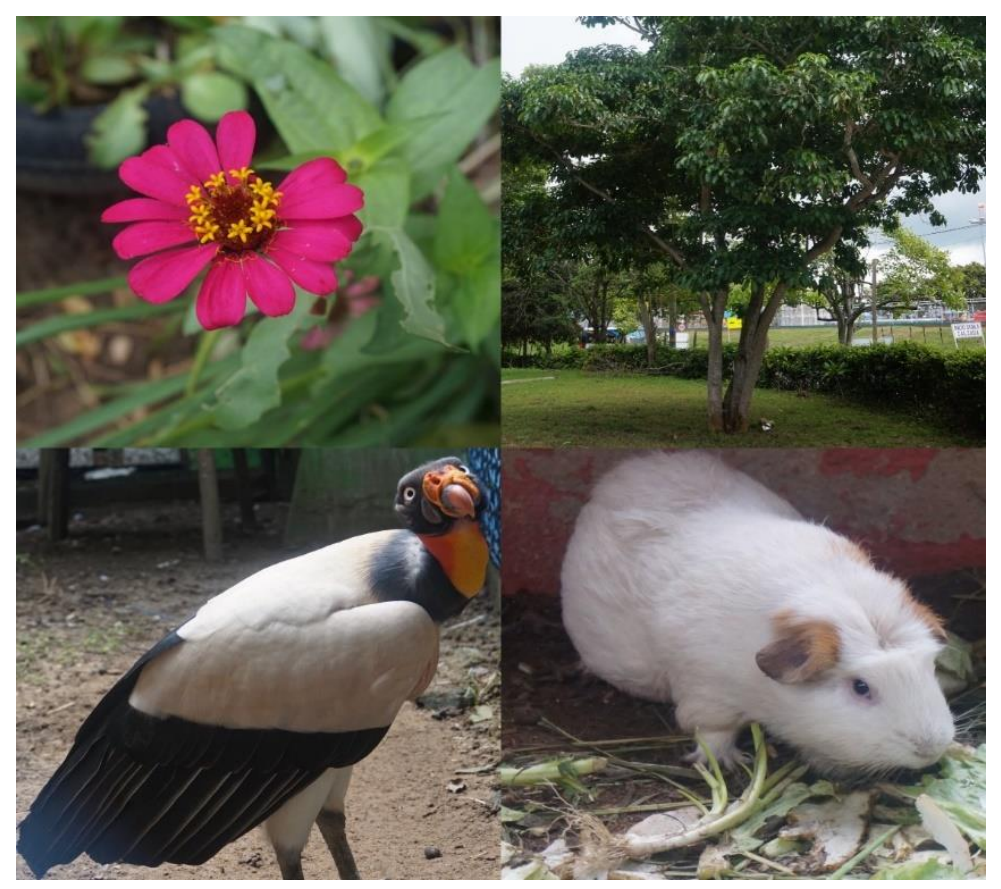

Figura 1. Collage de diferentes especies del Parque Biotemático Megua.

Fuente: Los autores. 


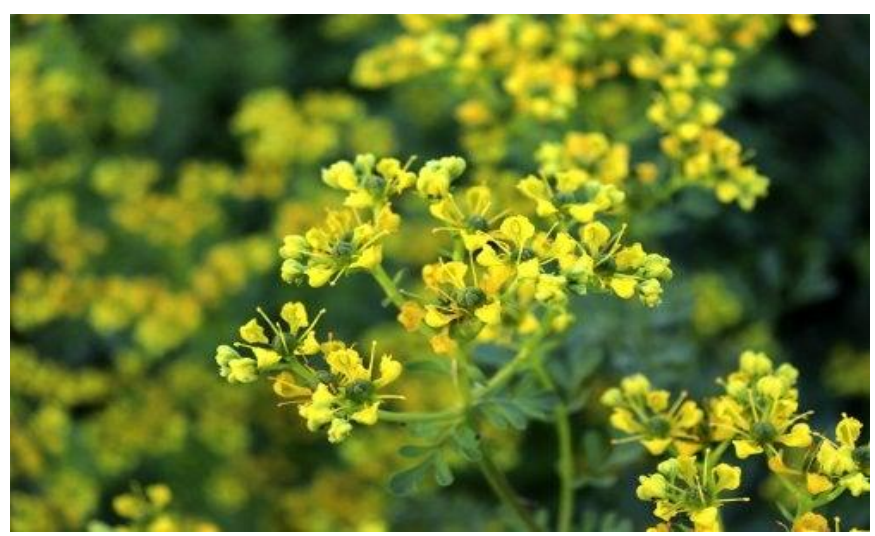

Figura 2. RUDA.

Fuente: Tomada de (Papa Pintor, 2020).

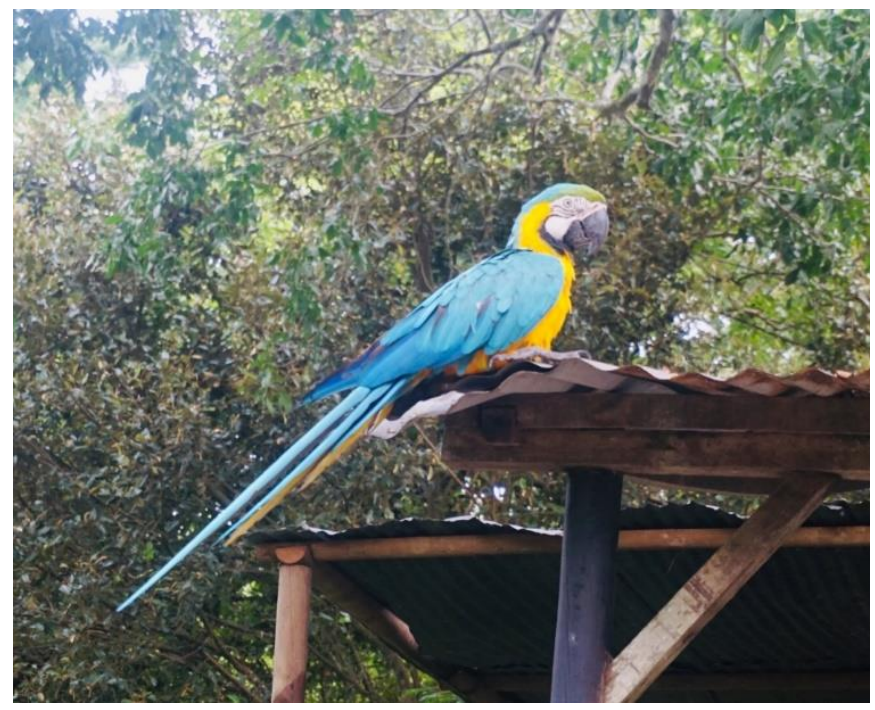

Figura 3. Guacamaya del parque Megua.

Fuente: Los autores.

\section{Características}

Tipo de planta: subhrub, forb/hierba

Esperanza de vida: perenne

Tiempo de florecimiento: verano

Tiempo de cosecha: otoño

Diámetro de flor: $2.0066 \mathrm{~cm}$

\section{Clasificación científica}

Reino: Vegetal

Género: ruta - rudas

Familia: rutaceae - rutáceas

Orden: Saphindales - Mangos, magnolias, margaritas y parientes

Clase: Magnoliopsida - magnolias, margaritas y parientes

Filo: Tracheophyta - plantas vasculares, monilophyta, tracheobionta.

\section{Requisito de condición}

Rusticidad, resistencia al frio: $-23.3333^{\circ} \mathrm{C}$

Suelo: Suelo arcilloso; buen drenaje; acido, neutro, alcalino

\section{Luz solar: Sol}

Usos y Hechos interesantes: Se puede plantar al aire libre, en parques, lugares escénicos o setos. Tiene muchos usos medicinales en la mayoría de los países latinoamericanos, la ruda se utiliza para cólicos abdominales, menstruales, las crisis histéricas, parásitos intestinales, hemorragias uterinas, la salmonelosis; para el uso externo se puede utilizar con compresas para los dolores musculares, malestar reumático, artritis, sarna, eczemas, pie de atleta, también sirve como calmante y antiespasmódicos y contra toxinas; ahuyenta insectos, elimina piojos y la conjuntivitis. En Honduras, Paraguay y Perú, aparte de sus usos medicinales también es muy utilizada para traer buena suerte en el amor, el dinero y protección contra maleficios, robos y enemigos; sacudiendo un ramito de ruda sobre su cuerpo o cargando un pedacito de la planta consigo para ahuyenta las malas energías. En Bolivia y Cuba la ruda molida en agua hirviendo sirve para el dolor de cabeza y cuando hay zumbido de oídos. Se debe tener precaución con su uso en el embarazo o lactancia. Tampoco es recomendable consumir en grandes dosis ya que puede causar daños en el hígado, cambios de humor, fiebre irritaciones e incluso la muerte.

\section{Guacamaya}

Nombre científico: Ara ararauna.

Nombre en inglés: Blue - and - yellow Macaw.

Otros nombres: Guacamayo azul y amarillo, Papagayo amarillo, Paraba azul amarillo.

Descripción: Es el representante característico de los guacamayos, loros con fuertes picos y largas colas. Mide entre 76 y $86 \mathrm{~cm}$ de largo y pesa de 900 a 1500 g. Tanto el macho como la hembra cuando son adultos son usualmente similares, su plumaje es de color azul en la parte superior, de color amarillo teñido de oro en el pecho y el vientre, la barbilla es de color azul oscuro y la frente verde. El pico es de color negro y las patas de color gris oscuro. La cara es de color blanco y llena de pequeñas plumas negras, volviéndose de color rosa en las aves excitadas.

Tiempo de vida: Es un ave que puede llegar a vivir unos 60 años aproximadamente. 


\section{Clasificación científica}

Reino: Animales - Animalia

Filo: Cordados - Chordata

Clase: Aves

Orden: Loros - Psittaciformes

Familia: Loros del nuevo mundo y África -

Psittacidae

Género: Guacamaya azul amarilla - Ara

Especie: Ara ararauna.

Habitad y distribución: Su área de distribución comprende desde Panamá hasta el noreste de Argentina pasando por toda la cuenca amazónica. Viven en hábitats variados que van desde bosque húmedo tropical, selva tropical a Sabana seca. Viven principalmente en bosques cercanos a los cauces de los ríos.

Comportamientos: Suelen vivir en parejas o en grupos pequeños, también se les suele ver en bandadas grandes de hasta unos 50 ejemplares aproximadamente, ruidosos animales que tienen como habito descansar largos periodos de tiempos y es capaz de interactuar con el ser humano.

Amenazas: Es cazado indiscriminadamente por coleccionistas y contrabandistas debido al gran mercado de venta de estos animales como mascotas de cautiverio, llegando a ser una especie de en peligro de extinción.

Reproducción: Forman parejas una sola vez. Anidan cada dos años entre agosto y enero, excavan agujeros en los troncos de los árboles y palmeras y la hembra normalmente pone dos o tres huevos, los cuales incuba durante unos 28 días y los polluelos dejan el nido en alrededor de 90 días después de la eclosión. El macho alimenta a la hembra durante este período y protege el nido de los intrusos. Los polluelos nacen sin plumas y ciegos y son alimentados por ambos padres con frutos y semillas regurgitados, durando en el nido durante tres meses. Incluso después las crías permanecen con los padres un máximo de un año y alcanzan la madurez sexual solo después de tres o cuatro años.

Alimentación: Su alimentación está basada en frutas, como el mango, semillas y los adquieren de los árboles o también la complementa con insectos, bayas, flores, tubérculos, vegetales.

Hechos interesantes: Ara es una onomatopeya del lenguaje Tupí, Ararauna también deriva del este lenguaje y significa loro grande y oscuro. Tupí que significa ave o guacamaya.

\subsection{Desarrollo de la aplicación}

El funcionamiento de la aplicación se muestra en el siguiente diagrama de flujo que aparece en la Figura 4. Ahora bien, se dispone a aclarar la información sobre la creación de la aplicación.

\subsubsection{Diseño}

\section{a. Boceto}

Hay mucho que decidir sobre este paso, el tema de color global de la aplicación, el estilo del encabezado, su modo de navegación, el ícono que lo representará en las tiendas y/o en las pantallas de inicio de los usuarios, su pantalla de bienvenida etc.

Al diseñar estos elementos, siempre es una buena idea mantener el aspecto algo alineado con cualquier diseño existente que la marca ya tenga y se identifique, como logotipos, colores, fuentes, entre otros.

En secuencia, en la Figura $5, \underline{6}$ y $\underline{7}$ se muestra los mockups correspondientes a la apariencia con la que se visualizará la aplicación, respectivamente a la página de inicio y HOME, plantilla de fauna y plantilla de flora.

El Home será la página más visitada y dará la primera impresión a los usuarios cuando descarguen la App. Debe mostrar las características más importantes, así como proporcionar una navegación fluida hacia las diferentes secciones de la aplicación.

\section{b. Contenido}

Es hora de agregar todo y cualquier cosa que se desee transmitir sobre el proyecto, es decir, todo lo relacionado con la Fauna y Flora de acuerdo con el Parque Biotemático Megua.

Se planea sólo ingresar este contenido directamente en el back - end de la app manualmente para así tener una mayor experiencia para brindar al cliente, dado que como el Parque es extenso, y como este carece de una buena cobertura de red Wi-Fi en algunas zonas del parque, la 


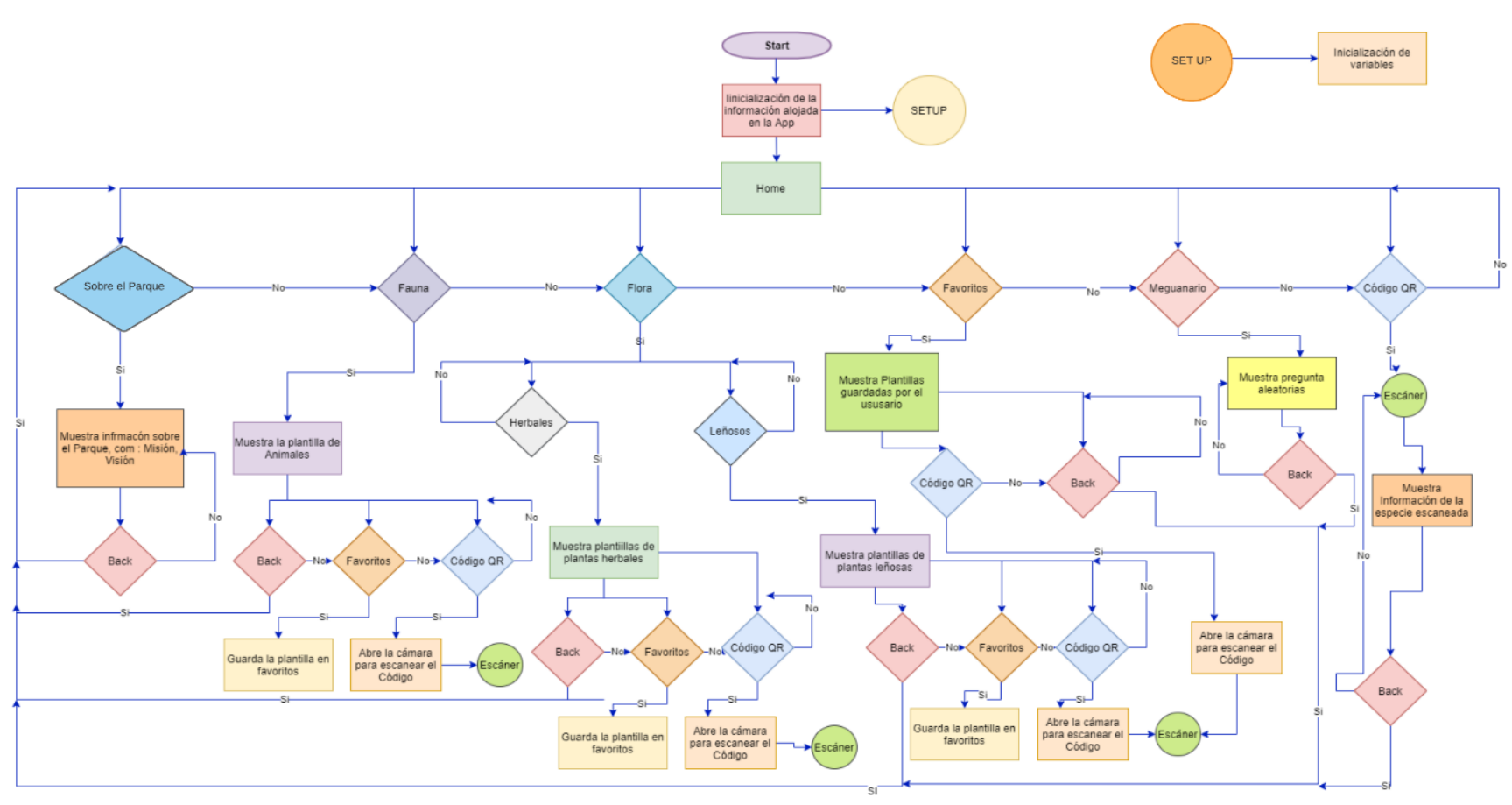

Figura 4. Flujograma de funcionamiento de la App.

Fuente: Los autores.

La imagen en alta definición de esta figura se encuentra al final del documento.

intención es que al inicio los usuarios finales, puedan descargar la aplicación con conexión a la red inalámbrica, y en su defecto toda la información la tendrán en su dispositivo para poder ofrecer una experiencia completa al usuario, y no depender de las peticiones que se tengan que realizar a un servidor posteriormente.

$$
\text { c. Add - ons: }
$$

Son complementos para agregar valor a la aplicación de acuerdo con las necesidades. Por ejemplo, para la sección de juegos, se utilizó una librería de Android, la cual realiza de acuerdo con las preguntas y respuestas bien colocadas, una aleatoriedad conforme a las preguntas, para que cada usuario responda diferentes interrogantes al momento de realizar el cuestionario, es decir, en diferente orden $y / o$ dificultad.

\section{d. Código QR}

La implementación de códigos QR en diferentes especies que se encuentren en el Parque les permiten a los visitantes leerlos desde sus dispositivos móviles y poder acceder a la información de dichas especies. La elección de este sobre el código de barras se realiza por ser una versión más actualizada, es de rápida respuesta, su diseño se puede personalizar y es de acceso libre.

El código QR, conocido como Quick Response en inglés, es un código de barras bidimensional cuadrado, en el cual se puede almacenar información en una matriz de puntos, con capacidad de almacenamiento de 7089 caracteres numéricos, 4296 caracteres alfanuméricos o 2953 bytes, siendo inmune a cierta distorsión de imágenes gracias a un sistema de corrección (COGNEX, 2020).

Estos códigos funcionan de manera muy simple, comenta (Rodríguez, 2020), solo con contar con un lector de códigos, en el dispositivo móvil se podrá escanear con la cámara de este el código que se desee; luego este último, redirecciona de manera inmediata hacia la información que se guardó en él, sea una página web, localización de GPS, conexión a red WIFI, descargar archivos, mostrar imágenes, entre otros.

Según (Sordo, 2020), estos códigos se forman con 4 elementos importantes:

1. Los identificadores, con los cuales cada versión de código QR se diferencia. 

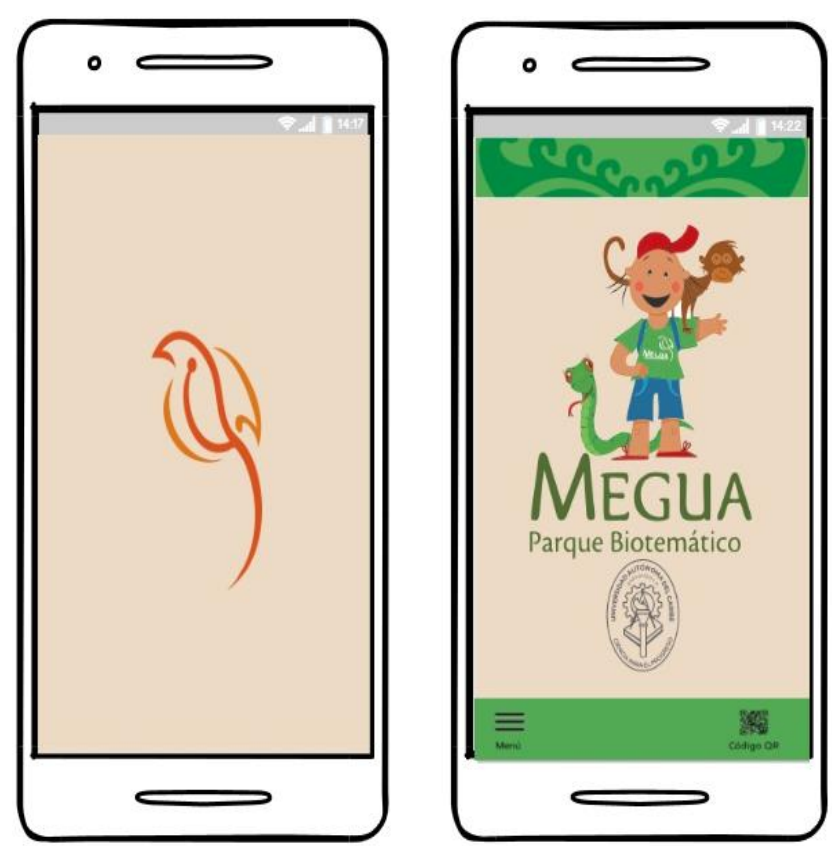

Figura 5. Mockup de inicio y HOME de la aplicación.

Fuente: Los autores.
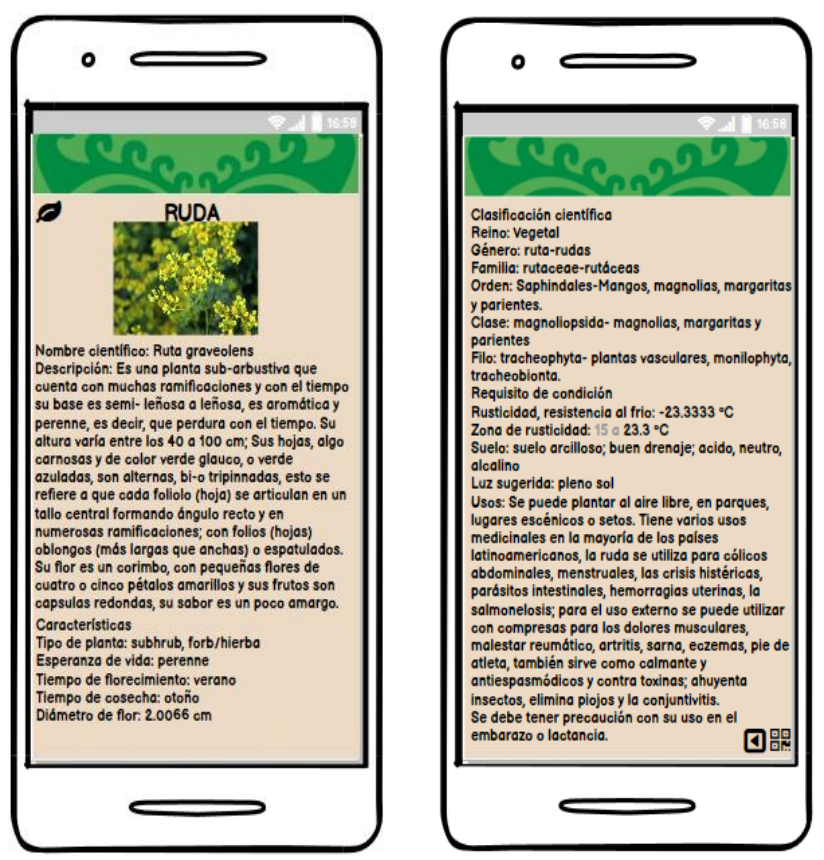

Figura 7. Mockup de Flora, (Herbal "RUDA").

Fuente: Los autores.

2. El formato, en el cual se brinda conocimiento acerca de su configuración.

3. Los datos, que serán específicos para cada código, dado a que se guarda la información y luego se despliega a el usuario después de ser escaneados.

4. Patrones requeridos; dice (Gingles, 30 cosas que debes saber de los códigos QR, 2016), se encuentra seccionado de la siguiente manera:
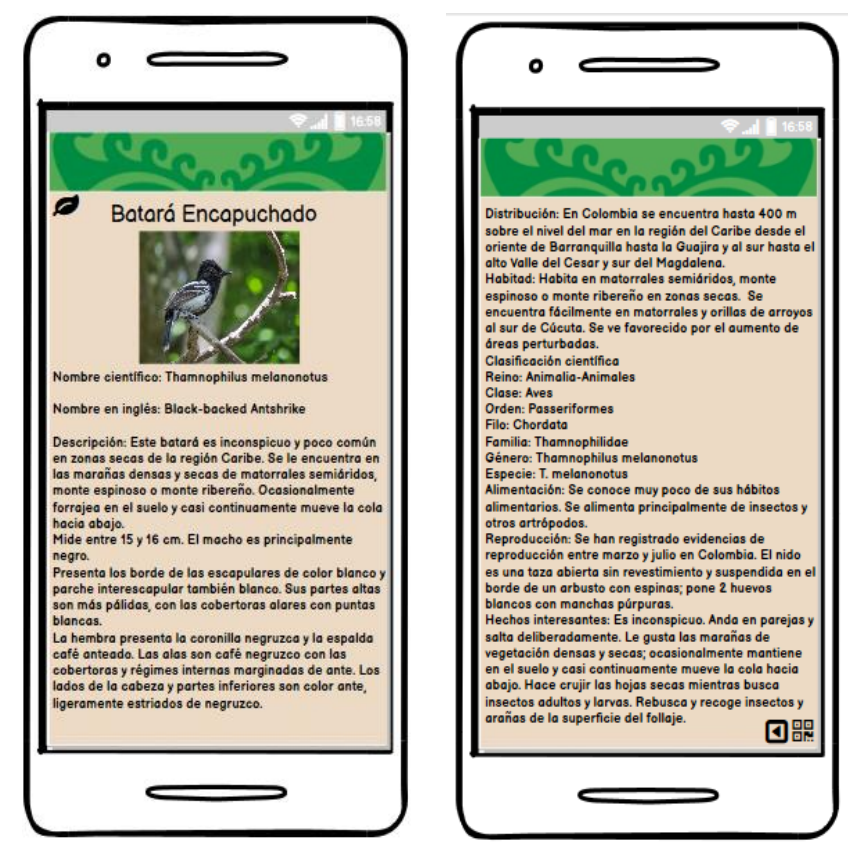

Figura 6. Mockup de Fauna (pájaro, "Batará Encauchado").

Fuente: Los autores.

- Posicionamiento: determina la dirección, permitiendo una lectura en $360^{\circ}$ de un código QR.

- Patrón de alineación: asegura que un código QR se pueda leer en el caso de que este impreso sobre una superficie curva o distorsionada debido al ángulo.

- Patrón de tiempo: permite que el decodificador determine el ancho de un código QR.

- Zona quieta: ayuda a aislar un código QR de su entorno.

Lo antes mencionado, se puede divisar mejor en la Figura 8, donde se diferencia con colores cada parte de la estructura del diseño de los códigos QR. Existen diferentes versiones de códigos QR, menciona (Gingles, 30 cosas que debes saber de los códigos QR, 2016), desde la versión $1(21 \times 21$ módulos) a la versión $40(177 \times 177$ módulos $)$. Cuantos más módulos haya en un código QR, más datos se podrán almacenar. Estas versiones se pueden diferenciar en la Figura 9.

Estos códigos pueden ser dinámicos o estáticos, diferenciándolos en que en el primero se puede modificar la información o contenido y el segundo no, además hay diferentes tipos, ente los cuales 


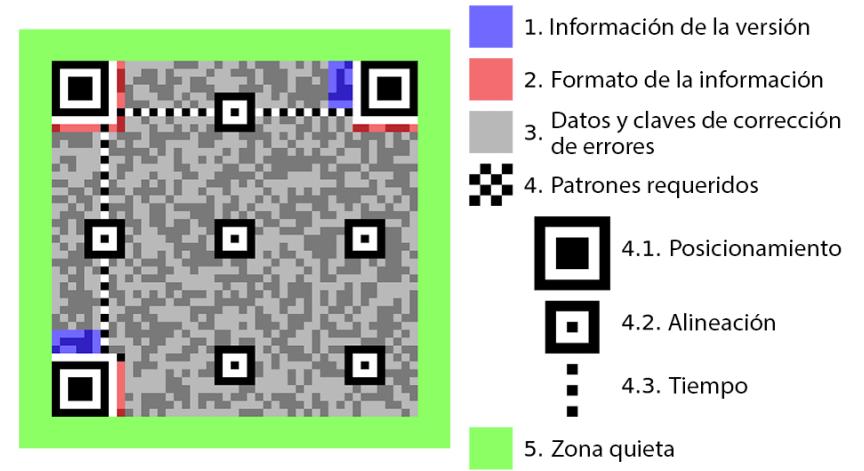

Figura 8. Estructura detrás del diseño de un código QR.

Fuente: Tomado de (Gingles, 30 cosas que debes saber de los códigos QR, 2016).

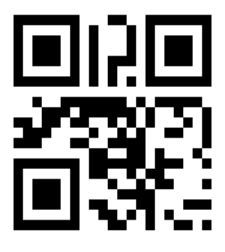

Version $1(21 \times 21)$

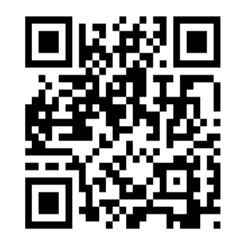

Version 3 (29×29)

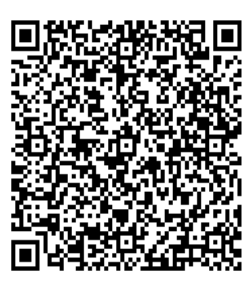

Version $10(57 \times 57)$
Figura 9. Versiones de un código QR.

Fuente: Tomado de (Gingles, 30 cosas que debes saber de los códigos QR, 2016).

menciona (Gingles, 30 cosas que debes saber de los códigos QR, 2016), se encuentran Micro QR, código iQR, SQRC y FrameQR.

\section{e. Complementos personalizados}

Después de reunir todas las piezas del proceso de creación de aplicaciones (concepto, diseño y tecnología), se alcanzará una versión que está casi lista para ser lanzada.

\section{Prueba de la Aplicación}

Se debe realizar un pedido de prueba, para verificar que el cliente final no tenga ningún inconveniente al momento de utilizar la aplicación y así probar todas las versiones que se planea publicar. En el caso de esta propuesta, la aplicación se desarrolló en plataforma Android; algunas de las pruebas a efectuar son las siguientes:

- Velocidad general de la app.

- No falle al realizar cualquier acción.

- Toda la funcionalidad / interacción responde a la tarea que se supone que debe realiza.

- El diseño es visualmente agradable y se adapta correctamente a todas las pantallas.
- La navegación es intuitiva y eficiente.

- El nombre de la aplicación / descripción se explique por sí mismo.

\section{Publicación de la Aplicación}

Una vez ya realizada todas las pruebas pertinentes y que todo esté funcionando como debería, es hora de pensar en la publicación.

Android ofrece diferentes opciones para distribuir aplicaciones, sin embargo, con más de mil millones de usuarios activos, Google sigue siendo la principal plataforma para apps con este sistema operativo.

\section{a. Listado de la información obligatoria}

Después de la creación de la aplicación en la consola de Google, se debe registrar la información que se mostrará en Google Play, ésta incluye información obligatoria como: título, descripción breve, descripción completa, activos gráficos, categoría, datos de contacto, política de privacidad.

\section{b. Archivo APK y app de lanzamiento}

Una vez completada la lista de la información obligatoria, se deberá cargar el archivo APK. El paquete de Android (Android Package Kit) contiene todos los elementos de la aplicación; continuando con, la guía en la consola de Google para implementar y publicar la app. Si ésta es aprobada, se lanzará aproximadamente 3 días hábiles.

\section{Resultados y discusión}

De acuerdo con el desarrollo de esta propuesta se logró recolectar a manera de inventario las especies de flora y fauna existentes en el parque.

Además, se espera que cuando finalice el desarrollo de la aplicación móvil, ésta sea un recurso más del parque que esté a disposición de los visitantes, siendo un recurso único, dado a que no existía con anterioridad. Asimismo, esperar que contribuya al posicionamiento y visualización del Parque, puesto a que, con ayuda de nueva tecnología, será un aporte para los senderos, los hará más atractivos a la vista, siendo una contribución ambiental y social que puede repercutir en un 
aumento de los recursos económicos para invertir posteriormente en éste.

También, se tomó como referencia dos aplicaciones, Picture This y Seek by iNaturalist, las cuales usan el poder de la tecnología con el reconocimiento de imagen para identificar las plantas, pájaros y animales que están alrededor y brindar información a los usuarios acerca de estas especies.

A diferencia de las aplicaciones antes mencionadas, la aplicación del Parque Biotemático Megua cuenta con un inventario e información propia, además de contar con un filtro de búsqueda para encontrar la información específica de la especie que se desee. Esto es algo con lo que aplicaciones como Picture This y Seek no cuentan.

Adicional, es una aplicación gratuita en su totalidad a comparación de las referenciadas previamente, ya que para acceder a algunas funciones en estas se debe desbloquear un servicio premium, realizando un pago anticipado para poder tener disponible las opciones de la aplicación en conjunto como por ejemplo, filtrar la búsqueda de información y lograr encontrar una especie en específico sin la necesidad de escanear, además de no contar con la opción de "favoritos", y tener a disposición posteriormente a las plantillas específicas que le han gustado al usuario y requieran un acceso rápido.

Por otra parte, la aplicación del Parque Biotemático Megua tendrá como entretenimiento al usuario, un cuestionario con orientación académica que pondrá a prueba los conocimientos obtenidos durante el recorrido de los senderos, brindado por el personal de apoyo o guías turísticos, algo con lo que las aplicaciones de Picture This y Seek no cuentan.

Por último, a diferencia de otros parques que han decidido realizar la instalación de códigos QR en las plantas, lo hacen irrumpiendo la sanidad de la flora. Por consiguiente, se decide buscar un método no invasivo para la instalación de estos códigos, el cual consiste en un método artesanal, colgándolos al rededor del árbol con un material orgánico, conocido por su durabilidad y resistencia, el cual se irá ajustando a la medida del tronco conforme la planta vaya creciendo.

\section{Conclusión y Trabajos futuros}

Para concluir, se instalaron 50 códigos QR, entre la fauna y flora en dos de los senderos más trascurridos del Parque; la instalación fue exitosa gracias al método de la instalación de los códigos con ayuda de los materiales orgánicos utilizados

El recurso de la información digital fue utilizado para los guías del parque haciendo más amena la información que les suministran a los visitantes del parque.

El meguanario o cuestionario académico, ha sido aceptado por los turistas del parque, sacando a flote habilidades como ejercicio de memoria, habilidades motrices, igualmente llevando consigo conceptos aprendidos del medio ambiente.

Se espera que generaciones futuras de estudiantes de ingeniería consideren seguir con la labor de crear tecnología para concientizar a las personas de cuidar nuestro ecosistema y medio ambiente.

Como recomendación, se puede realizar mejoras en esta propuesta, efectuando cambios como el de la lectura de código por inteligencia artificial, para tener reconocimiento de cualquier especie de manera inmediata.

También, se podría agregar contenido visual de las comunidades aledañas sobre los conocimientos de las especies del parque y sus utilidades caseras o habituales, lo anterior con respecto a las plantas herbales.

\section{Agradecimientos}

Se desea expresar agradecimiento por el apoyo brindado, a los Ingenieros de Sistemas Rubén Cabrera y Alberto Castaño.

\section{Referencias}

CARACOL RADIO. (20 de enero de 2020). Cortolima lanza plan de identificación de árboles. Ibagué, Tolima, Colombia. Obtenido de https://caracol.com.colemisora/2020/01/21/ibag ue/1579574368_803972.html 
COGNEX. (2020). Introduction to industrial barcode reading. Understand the inner workings of $1 D$ an $2 D$ codes, printing and making methods, and types of barcode readers. Obtenido de www.cognex.com

Gingles, L. (2 de Septiembre de 2016). Obtenido de uQR.me: $\quad$ https://uqr.meles/blog/cosas-quedebes-saber-sobre-codigos-grl

Papa Pintor, Y. (22 de Enero de 2020). Obtenido de https://mejorconsalud.com/propiedades-de-larudal

Rodríguez, A. (18 de Agosto de 2020). GoDaddy. Obtenido de https:/les.godaddy.com/blog/quees-un-codigo-qr-y-como-funcional

Shandilya, P. (12 de Febrero de 2019). Árboles del jardín de Lodhi etiquetados con código $Q R$. INDIATODAY. Obtenido de https://www.indiatoday.in/mailtoday/story/lodhi-garden-trees-tagged-with-qrcode-1453825-2019-02-12

Sordo, A. I. (25 de Agosto de 2020). HubSpot. Obtenido de https://blog.hubspot.es/marketing/como-crearun-codigo-qr 
Gutiérrez Polo et al. (2020). Revista Sextante, 23, pp. 37 - 47, 2020.

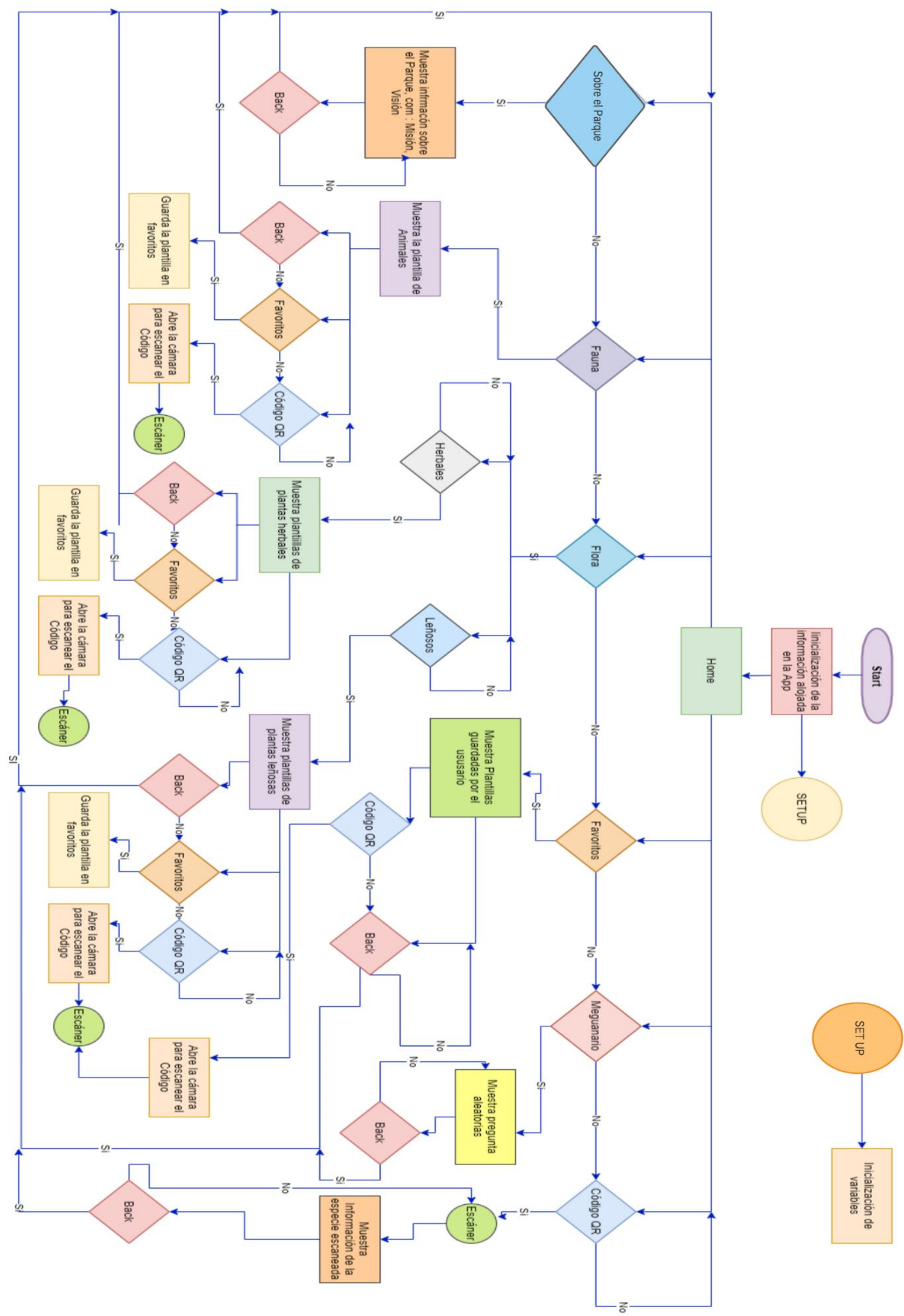

